\title{
Hybrid System Based Adaptive Control for the Nonlinear HVAC System
}

\author{
Ming-Li Chiang and Li-Chen Fu
}

\begin{abstract}
An adaptive controller is designed for the nonlinear MIMO heating, ventilating, and air conditioning (HVAC) system. The designed controller has the ability to adapt to the slowly time varying load change of thermal space and maintains good tracking performance for temperature and humidity ratio. Moreover, we integrate a supervisory switching logic into this system to adjust the $\mathrm{CO}_{2}$ concentration of thermal space and the whole closed loop system is modelled as a hybrid system. The obtained control system shows robustness and effectiveness and simulation results are provided to illustrate the control performance.
\end{abstract}

\section{INTRODUCTION}

Occupant comfort and energy efficiency are two primary goals of control strategies for the heating, ventilating, and air conditioning (HVAC) systems. As indicated in [1], HVAC systems for buildings are major consumers of electrical energy through the world. Temperature, humidity ratio and $\mathrm{CO}_{2}$ concentration are the quantitative indices of comfort in a room. To control such systems efficiently and effectively with dynamic interactions and disturbances so as to conserve energy while maintaining the desired thermal comfort level requires more than a conventional methodology. Classical HVAC control techniques such as the ON/OFF controllers (thermostats) and the proportional-integral-derivative (PID) controllers are still very popular because of their low cost. However in the long run, these controllers are expensive since they operate at a very low-energy efficiency. With increasing complexity of modern HVAC systems, how to control and optimize the operation with guaranteed performance, stability and reliability becomes a challenging issue. In fact, the air conditioning process is highly nonlinear and the interaction between temperature and humidity is significant. A nonlinear HVAC model which includes dynamics of temperature and humidity ratio is proposed in [2] where an observer to estimate the thermal load and moisture load is designed. The controller with load estimator can achieve the desired performance while the value of loads are not the designed one. In that paper, the load dynamics are assumed to be simple ones, that is, the loads are assumed to be constants. In [3], feedback linearization technique is applied to the same model. In [4], the actuator's dynamics are considered and the feedback linearization approach is adopted to design the controller. The thermal load is treated as a measurable disturbance and is compensated by the feedback controller. But the humidity ratio of thermal space

Ming-Li Chiang is with the Dept. of Electrical Engineering, National Taiwan University, Taipei, Taiwan d1921005@ee.ntu.edu.tw

$\mathrm{Li}-\mathrm{Chen} \mathrm{Fu}$ is with the Department of Electrical Engineering, and the Department of Computer Science and Information Engineering, National Taiwan University, Taipei, Taiwan lichen@ntu.edu.tw is not controlled since the authors chose different output function. In [5], a decentralized nonlinear adaptive controller consists of a fuzzy feedback controller and a frequencydomain adaptive compensator designed in Fourier space is proposed. The control scheme is capable of dealing with the varying thermal loads and is with short setting.

In this paper, we design an adaptive controller for the nonlinear MIMO HVAC system which is modelled to have some unknown parameters, to achieve robust and good heating, ventilating, and air conditioning performance. These slowly time varying unknown parameters are the thermal heat load and moisture load. In most of the related literatures, the values of the loads are treated as constants. Therefore, the controller proposed here should be more robust, more practical, and still achieving satisfactory performance. In particular, the system with the adaptive controller can operate effectively in the presence of dynamic interactions and disturbances while maintaining the desired thermal comfort level. Adaptive control for nonlinear systems has received a significant research attention and has evolved as a powerful methodology for nonlinear systems with parametric uncertainties. Feedback linearization approach proposed in [6] enhances the robustness to the failure of exact linearization. Many applications of nonlinear adaptive control have been presented in the past few years. In [7], the nonlinear adaptive controller based on feedback linearization is applied to the electro-hydraulic servomechanism. The SISO nonlinear system has a strong relative degree of two and trivial zero dynamics. In [8], adaptive control for nonlinear system are combined with the neural network approach and the nonlinear systems are assumed to have the normal form. This approach has the advantage with increasing computation speed and no need to analyze the complex nonlinear behavior of the system. The HVAC system considered in our paper is two-input, twooutput, and has no relative degree. We employ the dynamic extension algorithm to synthesize the feedback controller for the system and then design an adaptive controller to track the desired temperature and humidity ratio.

The remainder of this paper is organized as follows: section II introduces operation process and the hybrid dynamic model of the HVAC system. In section III, feedback linearization via dynamic extension is applied and a nonadaptive controller is designed to achieve the asymptotical stability for the tracking error. Then, we introduce some parametric uncertainties to replace the ideal model and derive the adaptive controller for the MIMO nonlinear plant. We also integrate a switching scheme to adjust the $\mathrm{CO}_{2}$ concentration. Section IV shows the simulation results and section $\mathrm{V}$ concludes this paper. 


\section{Hybrid System Model For the HVAC System AND PROBLEM FORMULATION}

\section{A. HVAC Model with Temperature and Humidity Ratio}

Air in the room is assumed to have an uniform temperature distribution and heat loss between components is neglected. Here we employ the model proposed in [2] which includes both temperature and humidity ratio. The system operates as shown in Fig. 1. Outdoor air enters the system at temperature $T_{0}$ and with volumetric flow rate $f_{r}(t)$. Air with temperature $T_{0}$ and flow rate $f_{r}(t)$ passes through the heat exchanger where an amount of heat is exchanged with the air. Since we have the assumption of perfect mixing, the air temperature inside and exiting the heat exchanger is $T_{2}(t)$, which represents the supply air temperature. After being cooled or heated in the heat exchanger, the air with temperature $T_{2}$ passes into the thermal space with the help of fan and air temperature of the thermal space is $T_{3}(t)$. The heat load in the room is denoted as $Q_{o}$. To save energy, typically $25 \%$ of the air is drawn out of the thermal space through the help of fan, and $75 \%$ of the air is recirculated to be mixed with the fresh air from outdoor.

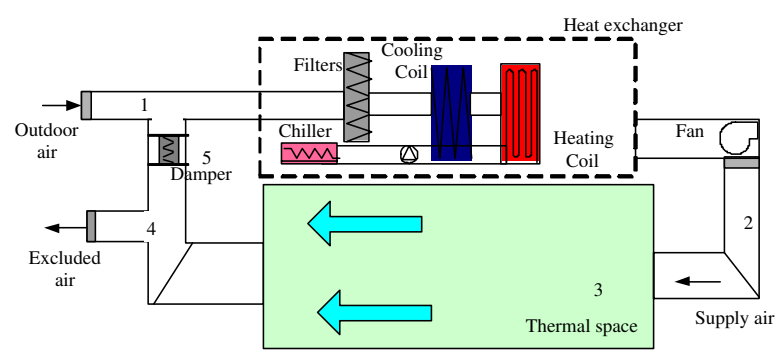

Fig. 1. Schematic layout of the HVAC system

Here, we assume the system is operating either with the cooling process and or with the heating process, but both models will be the same except the signs which can be either positive or negative. Control inputs to the system are the pumping rate $(\mathrm{gpm})$ of cold water from chiller to heat exchanger and the air flow rate $\left(f_{r}\right)$ using the variable speed fan. Notations and parameter values used in the dynamic model and simulations here are the same as those in [2] and are given in Table I.

From energy conservation principle, the dynamic equations of the HVAC system are given by

$$
\begin{aligned}
\dot{T}_{3}= & \frac{60 f_{r}}{V_{s}}\left(T_{2}-T_{3}\right)-\frac{60 h_{f g} f_{r}}{c_{p} V_{s}}\left(W_{s}-W_{3}\right) \\
& +\frac{1}{(1-\mu) \rho_{a} c_{p} V_{s}}\left(Q_{o}-h_{f g} M_{o}\right) \\
\dot{W}_{3}= & \frac{60 f_{r}}{V_{s}}\left(W_{s}-W_{3}\right)+\frac{M_{o}}{\rho_{a} V_{s}} \\
\dot{T}_{2}= & \frac{60 f_{r}}{V_{h e}}\left(T_{3}-T_{2}\right)+\frac{60(1-\mu) f_{r}}{V_{h e}}\left(T_{0}-T_{3}\right) \\
& -\frac{60 h_{w} f_{r}}{c_{p} V_{h e}}\left((1-\mu) W_{0}+\mu W_{3}-W_{s}\right)-6000 \frac{\mathrm{gpm}}{\rho_{a} c_{p} V_{h e}} .
\end{aligned}
$$

TABLE I

HVAC SYSTEM VARIABLES AND PARAMETERS

\begin{tabular}{|l||l|}
\hline$c_{p}$ & Specific heat of air at constant pressure $0.24\left(\mathrm{btu} / \mathrm{lb} \cdot{ }^{\circ} \mathrm{F}\right)$ \\
\hline$\rho_{a}$ & Air mass density $0.074\left(\mathrm{lb} / \mathrm{ft}^{3}\right)$ \\
\hline$V_{h e}$ & Volume of heat exchanger $60.75\left(\mathrm{ft}^{3}\right)$ \\
\hline$V_{s}$ & Volume of thermal space $58464\left(\mathrm{ft}^{3}\right)$ \\
\hline$W_{0}$ & Humidity ratio of outdoor air $0.018(\mathrm{~b} / \mathrm{lb})$ \\
\hline$W_{s}$ & Humidity ratio of supply air $0.0070(\mathrm{lb} / \mathrm{lb})$ \\
\hline$W_{3}$ & Humidity ratio of thermal space $(\mathrm{lb} / \mathrm{lb})$ \\
\hline$T_{0}$ & Temperature of outdoor $85\left({ }^{\circ} \mathrm{F}\right)$ \\
\hline$T_{2}$ & Temperature of supply air $\left({ }^{\circ} \mathrm{F}\right)$ \\
\hline$T_{3}$ & Temperature of thermal space $\left({ }^{\circ} \mathrm{F}\right)$ \\
\hline$M_{o}$ & Moisture load $166.06(\mathrm{lb} / \mathrm{hr})$ \\
\hline$Q_{o}$ & Sensible heat load $289897.52(\mathrm{btu} / \mathrm{hr})$ \\
\hline$h_{w}$ & Enthalpy of liquid water $(\mathrm{J} / \mathrm{lb})$ \\
\hline$h_{f g}$ & Enthalpy of water vapor $(\mathrm{J} / \mathrm{lb})$ \\
\hline$f_{r}$ & Volumetric flow rate of air $\left(\mathrm{cfm}=\mathrm{ft}{ }^{3} / \mathrm{min}\right)$ \\
\hline$f_{r 0}$ & Initial of volumetric flow rate of air $4250(\mathrm{cfm})$ \\
\hline $\mathrm{gpm}$ & Flow rate of chilled water $(\mathrm{gal} / \mathrm{min})$ \\
\hline$\mu$ & Recirculation rate of air in system $(75 \%)$ \\
\hline$C_{s}$ & CO ${ }_{2}$ concentration of thermal space $(\mathrm{ppm})$ \\
\hline
\end{tabular}

and the state equations can be described as

$$
\begin{aligned}
\dot{x}_{1}= & u_{1} \alpha_{1}\left(x_{3}-x_{1}\right)-u_{1} \alpha_{2}\left(W_{s}-x_{2}\right) \\
& +\alpha_{3}\left(Q_{o}-h_{f g} M_{o}\right) \\
\dot{x}_{2}= & u_{1} \alpha_{1}\left(W_{s}-x_{2}\right)+\alpha_{4} M_{o} \\
\dot{x}_{3}= & u_{1} \beta_{1}\left(x_{1}-x_{3}\right)+(1-\mu) u_{1} \beta_{1}\left(T_{0}-x_{1}\right) \\
& -u_{1} \beta_{3}\left((1-\mu) W_{0}+\mu x_{2}-W_{s}\right)-6000 u_{2} \beta_{2} \\
y= & {\left[\begin{array}{ll}
h_{1}(x) & h_{2}(x)
\end{array}\right]^{T}=\left[\begin{array}{ll}
x_{1} & x_{2}
\end{array}\right]^{T} }
\end{aligned}
$$

where

$$
\begin{aligned}
& u_{1}=f_{r}, u_{2}=\mathrm{gpm}, x_{1}=T_{3}, x_{2}=W_{3}, x_{3}=T_{2} \\
& \alpha_{1}=\frac{60}{V_{s}}, \alpha_{2}=\frac{60 h_{f g}}{c_{p} V_{s}}, \alpha_{3}=\frac{1}{(1-\mu) \rho_{a} c_{p} V_{s}}, \alpha_{4}=\frac{60}{\rho_{a} V_{s}} \\
& \beta_{1}=\frac{60}{V_{h e}}, \beta_{2}=\frac{1}{\rho_{a} c_{p} V_{h e}}, \beta_{3}=\frac{60 h_{w}}{c_{p} V_{h e}}
\end{aligned}
$$

This is a bilinear system which is homogeneous in the state. Heat load $Q_{o}$ and moisture load $M_{o}$ are usually not measurable and hence will be regarded as unknown parameters. In [2], the authors develop an observer for the system to obtain the estimation of $Q_{o}$ and $M_{o}$. Now, consider the actuator dynamics for the control inputs, namely the valve dynamic as in [4]

$$
u_{1}=\frac{k_{1}}{1+\tau_{1} s} z_{1}, \quad u_{2}=\frac{k_{2}}{1+\tau_{2} s} z_{2}
$$

with $u=\left[\begin{array}{ll}u_{1} & u_{2}\end{array}\right]^{T}$ be the control signal applied to the plant and $z=\left[\begin{array}{ll}z_{1} & z_{2}\end{array}\right]^{T}$ the input signals applied to the actuator. Hence, we derive an augmented state space model with the new state vector $x:=\left[\begin{array}{lllll}x_{1} & x_{2} & x_{3} & u_{1} & u_{2}\end{array}\right]^{T}:=\left[\begin{array}{lllll}x_{1} & x_{2} & x_{3} & x_{4} & x_{5}\end{array}\right]^{T}$, and input signal $z=\left[\begin{array}{ll}z_{1} & z_{2}\end{array}\right]^{T}$, so that the system model now 
becomes

$$
\begin{aligned}
\dot{x} & =f(x)+g(x) z=f(x)+g_{1}(x) z_{1}+g_{2}(x) z_{2} \\
& =\left[\begin{array}{l}
a_{1}(x) \\
a_{2}(x) \\
a_{3}(x) \\
a_{4}(x) \\
a_{5}(x)
\end{array}\right]+\left[\begin{array}{cc}
0 & 0 \\
0 & 0 \\
0 & 0 \\
\frac{k_{1}}{\tau_{1}} & 0 \\
0 & \frac{k_{2}}{\tau_{2}}
\end{array}\right] z \\
y & =h(x)=\left[\begin{array}{ll}
x_{1} & x_{2}
\end{array}\right]^{T}
\end{aligned}
$$

where

$$
\begin{aligned}
a_{1}(x)= & {\left[\alpha_{1}\left(x_{3}-x_{1}\right)-\alpha_{2}\left(W_{s}-x_{2}\right)\right] u_{1}+\alpha_{3}\left(Q_{0}-h_{f g} M_{0}\right) } \\
a_{2}(x)= & \gamma_{1} u_{1}+\alpha_{3}\left(Q_{0}-h_{f g} M_{0}\right) \\
= & \alpha_{1}\left(W_{s}-x_{2}\right) u_{1}+\alpha_{4} M_{0} \\
a_{3}(x)= & \gamma_{2} u_{1}+\alpha_{4} M_{0} \\
= & {\left[\beta_{1}\left(x_{1}-x_{3}\right)+(1-\mu) \beta_{1}\left(T_{0}-x_{1}\right)\right] u_{1} } \\
& +\left[-\beta_{3}\left((1-\mu) W_{0}+\mu x_{2}-W_{s}\right)\right] u_{1}-\left[6000 \beta_{2}\right] u_{2} \\
:= & \gamma_{3} u_{1}+\gamma_{4} u_{2} \\
a_{4}(x)= & -\frac{u_{1}}{\tau_{1}} \text { and } a_{5}(x)=-\frac{u_{2}}{\tau_{2}}
\end{aligned}
$$

\section{B. Hybrid System Model for the HVAC System}

The quantitative indices of comfort in the room are temperature, humidity ratio, and $\mathrm{CO}_{2}$ concentration. In fact, the circulated air which contains too much $\mathrm{CO}_{2}$ will affect the work efficiency of people. Now, we consider the $\mathrm{CO}_{2}$ model and then propose a hybrid system model for the HVAC system. From the mass balance equation, the average $\mathrm{CO}_{2}$ concentration $C_{s}$ in the room can be represented as

$$
\dot{C}_{s}=\frac{C_{q}}{V_{s}}+(1-\mu)\left(C_{i}-C_{o}\right)
$$

where $C_{q}$ is the amount of $\mathrm{CO}_{2}$ generated in the room (normally from people entering the room), $C_{i}$ is the $\mathrm{CO}_{2}$ concentration of inlet air, $C_{o}$ is the $\mathrm{CO}_{2}$ concentration of air leaving the room, and $(1-\mu)$ is the air exchange rate. Assume that the air in the room is well-mixed and $C_{i}$ is a constant, then $C_{o}=C_{s}$ and the equation can be written as

$$
\dot{C}_{s}^{\prime}=\frac{C_{q}}{V_{s}}-(1-\mu) C_{s}^{\prime}
$$

where $C_{s}^{\prime}=C_{i}-C_{s}$. We can use nonlinear control of $(1-\mu)$ to adjust the $\mathrm{CO}_{2}$ concentration at all operating points. The value of $C_{q}$ is dependent on the number and the physical state of people in the room and there are some reference data in the ASHRAE standard. Instead of controlling $C_{S}$ to a desired value, we define three levels of $\mathrm{CO}_{2}$ concentration in the room and adjust the value of $\mu$ according to the level at which $C_{s}$ is located. That is, classify the $\mathrm{CO}_{2}$ concentration into three intervals $\left\{\mathrm{CO}_{2} \mathrm{Low}, \mathrm{CO}_{2} \mathrm{Med}, \mathrm{CO}_{2} \mathrm{High}\right\}$ and use the supervisory controller $S$ to decide the corresponding value of $\mu \in\left\{\mu_{1}, \mu_{2}, \mu_{3}\right\}$. Thus, the $\mathrm{CO}_{2}$ concentration is modelled and adjusted by discrete event systems theory. The HVAC system can be modelled as a hybrid system [9] which contains continuous states $x$ and discrete states $\mu$. The continuous dynamics in this system is as in (2) and the discrete dynamics is defined with the supervisory switching logic which will be designed in next section. Thus we have the hybrid system model for the HVAC system as follows:

$$
\begin{aligned}
& \text { Continuous dynamics: } \\
& \quad \begin{array}{l}
\dot{x}=f(x, \mu)+g z, \quad \mu \in\left\{\mu_{1}, \mu_{2}, \mu_{3}\right\} \\
y=h(x)
\end{array} \\
& \text { Discrete dynamics: } \\
& \quad \mu=\mu_{i}, \quad i \in\{1,2,3\} \text { assigned by } S
\end{aligned}
$$

We will use the continuous adaptive state feedback controller to control the value of $y$ and the discrete event controller $S$ to decide the value of $\mu$ and thus the $\mathrm{CO}_{2}$ concentration $C_{s}$ will be adjusted. Our control object is to track the desired temperature and humidity ratio, and construct a supervisor to keep the $\mathrm{CO}_{2}$ concentration low.

\section{Supervisory AdAPTIVE CONTROL FOR THE NONLINEAR MIMO HVAC SYSTEM}

In this section, we apply the feedback linearization technique [10] to the nonlinear HVAC system. Assume the values of loads $Q_{0}$ and $M_{0}$ are pre-specified constants and we design the non-adaptive dynamic state feedback controller for the HVAC system. Then, the adaptive controller is designed to cope with the slowly time varying unknown $M_{o}, Q_{o}$. We also integrate a supervisory switching mechanism which aim to keep the $\mathrm{CO}_{2}$ concentration in the HVAC system low.

\section{A. Feedback Linearization via Dynamic Extension for the HVAC System}

To reduce the nonlinear system to an aggregate of independent single-input, single-output channels, which is called the noninteracting control problem, we differentiate the outputs $y_{i}(t)$ until the inputs appear. The intuitive concept of relative degree is the smallest number of times that the output have to differentiate such that at least one of the inputs appears in $y_{i}^{\left(r_{i}\right)}$. For the 2-input, 2-output systems of the form (2), the relative degree is defined as $r=\left\{r_{1}, r_{2}\right\}$ which satisfies (i) $L_{g_{j}} h_{i}=L_{g_{j}} L_{f} h_{i}=\ldots=L_{g_{j}} L_{f}^{\left(r_{i}-2\right)} h_{i}=0$, for all $j=1,2$, $i=1,2$, and (ii) the decoupling matrix

$$
A(x)=\left[\begin{array}{cc}
L_{g_{1}} L_{f}^{r_{1}-1} h_{1} & L_{g_{2}} L_{f}^{r_{1}-1} h_{1} \\
L_{g_{1}} L_{f}^{r_{2}-1} h_{2} & L_{g_{2}} L_{f}^{r_{2}-1} h_{2}
\end{array}\right]
$$

is nonsingular near the equilibrium point $x=x^{e}$. Note that $L_{f} h_{i}$ stands for the Lie derivative of $h_{i}$ with respect to $f$. The definition of relative degree will lead to

$$
\left[\begin{array}{l}
y_{1}^{\left(r_{1}\right)} \\
y_{2}^{\left(r_{2}\right)}
\end{array}\right]=\left[\begin{array}{c}
L_{f}^{r_{1}} h_{1} \\
L_{f}^{r_{2}} h_{2}
\end{array}\right]+A(X)\left[\begin{array}{l}
z_{1} \\
z_{2}
\end{array}\right]
$$

and thus the feedback control law

$$
z=-A(x)^{-1}\left[\begin{array}{c}
L_{f}^{r_{1}} h_{1} \\
L_{f}^{r_{2}} h_{2}
\end{array}\right]+A(x)^{-1} v
$$

will yield the closed-loop decoupled, linear system

$$
\left[\begin{array}{l}
y_{1}^{\left(r_{1}\right)} \\
y_{2}^{\left(r_{2}\right)}
\end{array}\right]=\left[\begin{array}{l}
v_{1} \\
v_{2}
\end{array}\right]
$$


Given the desired output $y_{d}=\left[\begin{array}{ll}y_{1 d}, & y_{2 d}\end{array}\right]^{T}$ and define the output error as $e=\left[e_{1}, e_{2}\right]^{T}:=\left[\left(y_{1}-y_{1 d}\right),\left(y_{2}-y_{2 d}\right)\right]^{T}$. Then, we can design the control

$$
v_{i}=y_{i d}^{\left(r_{i}\right)}-c_{i 1} e_{i}^{\left(r_{i}-1\right)}-\cdots-c_{i r_{i}} e_{i}
$$

such that the error equation becomes

$$
e_{i}^{\left(r_{i}\right)}+c_{i 1} e_{i}^{\left(r_{i}-1\right)}+\cdots+c_{i r_{i}} e_{i}=0
$$

The coefficients $c_{i j}, j=1,2, \ldots, r_{i}$, are chosen so that $s^{r_{i}}+c_{i 1} s^{r_{i}-1}+\cdots+c_{i r_{i}}$ is a Hurwitz polynomial to meet the desired performance specification such as transient response or steady state error.

For the HVAC system (2), we have $r=\{2,2\}$, but the matrix

$$
A(x)=\left[\begin{array}{ll}
\gamma_{1} k_{1} / \tau_{1} & 0 \\
\gamma_{2} k_{1} / \tau_{1} & 0
\end{array}\right]
$$

is singular. Thus, the system has no relative degree. To achieve the relative degree and noninteracting control, we resort to the dynamic extension algorithm [10] to incorporate the dynamic state feedback into this system. Set $z_{1}=\psi_{1}$, $\dot{\psi}_{1}=\zeta_{1}$, and $z_{2}=\zeta_{2}$. Define the new augmented state as $\tilde{x}=\left[x, z_{1}\right]^{T} \in \mathbb{R}^{6}$ and the composed system will be

$$
\begin{aligned}
\dot{\tilde{x}} & =\tilde{f}(\tilde{x})+\tilde{g}_{1}(\tilde{x}) \zeta_{1}+\tilde{g}_{2}(\tilde{x}) \zeta_{2} \\
y & =h(x)
\end{aligned}
$$

where $\tilde{f}=\left[\begin{array}{llll}\tilde{a}_{1}, & \tilde{a}_{2}, \ldots, \tilde{a}_{6}\end{array}\right]^{T}$ is equal to $f$ except the term $\tilde{a}_{4}=a_{4}+\frac{k_{1}}{\tau_{1}} z_{1}$ and $\tilde{a}_{6}=0$. Moreover, the vector field $\tilde{g}=\left[\begin{array}{ll}\tilde{g}_{1} & \tilde{g}_{2}\end{array}\right]$, where $\tilde{g}_{1}=\left[\begin{array}{llllll}0, & 0, & 0, & 0, & 0, & 1\end{array}\right]^{T}$ and $\tilde{g}_{2}=$ $\left[0,0,0,0, \frac{k_{2}}{\tau_{2}}, 0\right]^{T}$. After calculation, we find the relative degree now becomes $\tilde{r}=\left\{\tilde{r_{1}}, \tilde{r_{2}}\right\}=\{3,3\}$ so that

$$
\left[\begin{array}{l}
y_{1}^{(3)} \\
y_{2}^{(3)}
\end{array}\right]=\left[\begin{array}{c}
L_{\tilde{f}}^{3} h_{1} \\
L_{\tilde{f}}^{3} h_{2}
\end{array}\right]+B(\tilde{x})\left[\begin{array}{l}
\zeta_{1} \\
\zeta_{2}
\end{array}\right]:=C(\tilde{x})+B(\tilde{x}) \zeta
$$

and the nonsingular decoupling matrix becomes,

$$
B(\tilde{x})=\left[\begin{array}{cc}
L_{\tilde{g}_{1}} L_{\tilde{f}}^{2} h_{1} & L_{\tilde{g}_{2}} L_{\tilde{f}}^{2} h_{1} \\
L_{\tilde{g}_{1}} L_{\tilde{f}}^{2} h_{2} & L_{\tilde{g}_{2}} L_{\tilde{f}}^{2} h_{2}
\end{array}\right]=\left[\begin{array}{cc}
\frac{\gamma_{1} k_{1}}{\tau_{1}} & \frac{\left(\alpha_{1} \gamma_{4} x_{4} k_{2}\right)}{\tau_{2}} \\
\frac{\gamma_{2} k_{1}}{\tau_{1}} & 0
\end{array}\right]
$$

Thus, design the control $\zeta=-B^{-1} C+B^{-1} v$ with $v=$ $\left[v_{1}, v_{2}\right]^{T}$ as discussed in (7), the output error will converge to zero and the system will be asymptotically stable.

Remark 1: Since the relative degree $\tilde{r}_{1}+\tilde{r}_{2}=6$ is equal to the number of states, the system has trivial zero dynamics and thus the internal stability and boundedness of states are guaranteed. If the system has relative degree $\tilde{r}_{1}+\tilde{r}_{2}=k<6$, we can obtain the internal dynamics by constructing the local coordinate transform with the function $\phi_{i}$ such that $L_{\tilde{g}_{j}} \phi_{i}=0$ for $j=1,2$ and $k+1 \leq i \leq 6$. The existence of the functions $\phi_{i}$ is guaranteed by the Frobenius theorem and the fact that constant vector fields $\tilde{g}_{1}, \tilde{g}_{2}$ are always involutive.

\section{B. Adaptive Control for the HVAC system}

The drawback of the feedback linearization approach is that the linearizing control law is based on exact cancellation of the nonlinear terms. If there is any uncertainty in the knowledge of the nonlinear functions $\tilde{f}, \tilde{g}$, the cancellation is not exact and the resulting input-output equation is not linear in practice. The value of heat thermal load $Q_{o}$ and that of moisture load $M_{o}$ are not measurable and are hence difficult to estimate. In this section, we will use adaptive control techniques [6] to solve this problem.

Define the values of $M_{0}$ and moisture load $Q_{0}$ as unknown parameters $\Theta^{*}:=\left[\theta_{1}^{*}=M_{o}, \theta_{2}^{*}=Q_{o}\right]^{T}$. The system is linear with respect to the vector field $\tilde{f}$ and the unknown constant parameter vector $\theta^{*}$, and thus we can rewrite (8) as

$$
\begin{aligned}
\dot{\tilde{x}} & =\sum_{i=1}^{2} \theta_{i}^{*} f_{i}(\tilde{x})+f_{0}+\tilde{g} \zeta \\
y_{i} & =h_{i}(x), \quad i=1,2 .
\end{aligned}
$$

The estimates of $\Theta^{*}$ is denoted as $\Theta$ and thus the estimate of the vector field $\tilde{f}$ is defined as $\hat{f}=\theta_{1} f_{1}+\theta_{2} f_{2}+f_{0}$. Thus, the Lie derivatives in our feedback control are replaced by the estimations as the follows:

$$
\begin{aligned}
L_{\hat{f}^{3}} h_{i} & :=\sum_{j=1}^{2} \sum_{k=1}^{2} \sum_{l=1}^{2} \frac{\partial}{\partial \tilde{x}}\left[\frac{\partial}{\partial \tilde{x}}\left(\frac{\partial h_{i}}{\partial \tilde{x}} f_{j}\right) f_{k}\right] f_{l} \theta_{j} \theta_{k} \theta_{l} \\
L_{\tilde{g}} L_{\hat{f}}^{2} h_{i} & :=\sum_{l=1}^{2} \sum_{j=1}^{2} \sum_{k=1}^{2} \frac{\partial}{\partial \tilde{x}}\left[\frac{\partial}{\partial \tilde{x}}\left(\frac{\partial h_{i}}{\partial \tilde{x}} f_{j}\right) f_{k}\right] \tilde{g}_{l} \theta_{j} \theta_{k}
\end{aligned}
$$

The ideal linearizing control law is replaced by

$$
\widehat{\zeta}=\widehat{B}^{-1}\left(-\left[\begin{array}{c}
L_{\hat{f}}^{3} h_{1} \\
L_{\hat{f}}^{3} h_{2}
\end{array}\right]+\widehat{v}\right)
$$

where $\widehat{B}$ is the estimate of $B$ and the implemented tracking law $\widehat{v}$ is of the following form

$$
\widehat{v_{i}}=y_{i d}^{(3)}+c_{1}\left(y_{i d}^{(2)}-L_{\hat{f}}^{2} h_{i}\right)+c_{2}\left(y_{i d}^{(1)}-L_{\hat{f}} h_{i}\right)+c_{3}\left(y_{i d}-y_{i}\right),
$$

$i=1,2$. Since in (10) $f_{1}$ and $f_{2}$ are not dependent on $\tilde{x}$, the terms $\left(\theta_{i} \theta_{j}, \theta_{i} \theta_{j} \theta_{k}\right)$ will not appear in $L_{\hat{f}}^{2} h_{i}, L_{\hat{f}}^{3} h_{i}, L_{\tilde{g_{j}}} L_{\hat{f}}^{2} h_{i}$, and thus the parameter vector we need to estimate is $\Theta=$ $\left[\begin{array}{ll}\theta_{1}, & \theta_{2}\end{array}\right]^{T} \in \mathbb{R}^{2}$. Substitute the control $\widehat{\zeta}$ into the system and define the parameter error $\Phi:=\Theta^{*}-\Theta$, then the error equation with the feedback control will become

$$
e^{(3)}+c_{1} e^{(2)}+c_{2} e^{(1)}+c_{3} e=\Xi \Phi
$$

where $\Xi=\left[\begin{array}{ll}\Xi_{11} & \Xi_{12} \\ \Xi_{21} & \Xi_{22}\end{array}\right]=\left[\begin{array}{l}\Xi_{1} \\ \Xi_{2}\end{array}\right] \in \mathbb{R}^{2 \times 2}$ and the terms on the right hand side are the mismatch between the linearizing law and the actual linearizing law as well as that between the tracking control $v$ and the actual tracking control $\hat{v}$. By computation we have

$$
\Xi_{i} \Phi=\left(\left[L_{\tilde{f}}^{3} h_{i}-L_{\hat{f}}^{3} h_{i}\right]+c_{1}\left[L_{\tilde{f}}^{2} h_{i}-L_{\hat{f}}^{2} h_{i}\right]+c_{2}\left[L_{\tilde{f}} h_{i}-L_{\hat{f}} h_{i}\right]\right)
$$

and $\Xi_{11}=\alpha_{3} h_{f g}\left(-\lambda_{1}+\alpha_{1} \tilde{a}_{4}+c_{1} \alpha_{1} u_{1}-c_{2}\right)+\alpha_{4}\left(\lambda_{2}+\right.$ $\left.\alpha_{2} \tilde{a}_{4}+c_{1} \alpha_{2} u_{1}\right), \Xi_{12}=\alpha_{3}\left(\lambda_{1}-\alpha_{1} \tilde{a}_{4}-c_{1} \alpha_{1} u_{1}+c_{2}\right), \Xi_{21}=$ 
$\alpha_{4}\left[\left(\alpha_{1} u_{1}\right)^{2}-2 \alpha_{1} \tilde{a}_{4}-c_{1} \alpha_{1} u_{1}+c_{2}\right], \quad \Xi_{22}=0$, and $\lambda_{1}=$ $\alpha_{1}\left[u_{1}^{2}\left(\alpha_{1}+\mu \beta_{1}\right)-\tilde{a}_{4}\right], \lambda_{2}=-2 \alpha_{1} \alpha_{2} u_{1}^{2}-\mu \beta_{3} \alpha_{1} u_{1}^{2}+\alpha_{2} \tilde{a}_{4}$.

For the case of relative degree 3 , we define the augmented error

$$
e_{i_{\text {aug }}}=b_{1} e_{i}^{(2)}+b_{2} e_{i}^{(1)}+b_{3} e_{i}
$$

such that the transfer function

$$
\left(b_{1} s^{2}+b_{2} s+b_{3}\right) /\left(s^{3}+c_{1} s^{2}+c_{2} s+c_{3}\right)
$$

is strictly positive real (SPR). Let

$$
e_{i_{\text {aug }}}=e_{i}+\left(\Theta^{T} L^{-1} \Xi_{i}^{T}-L^{-1} \Theta^{T} \Xi_{i}^{T}\right), \quad i=1,2
$$

where the polynomial

$$
L(s)=s^{(3)}+c_{1} s^{(2)}+c_{2} s^{(1)}+c_{3}
$$

is chosen to be Hurwitz and note that $e_{i}=L^{-1}(s) \Phi^{T} \Xi_{i}^{T}$. From the fact that $\Theta^{*}$ is a constant vector, we can obtain the error equation for adaptation, i.e., $e_{\text {iaug }}=\Phi^{T} L^{-1} \Xi_{i}^{T}$ and define $L^{-1} \Xi_{i}^{T}=\xi_{i}^{T} \in \mathbb{R}^{2 \times 1}$, then

$$
e_{\text {aug }}=\left[\begin{array}{l}
e_{1_{\text {aug }}} \\
e_{2_{\text {aug }}}
\end{array}\right]=\left[\begin{array}{l}
\xi_{1} \\
\xi_{2}
\end{array}\right] \Phi:=\xi \Phi
$$

Hence, we can use the normalized gradient adaptive law

$$
\dot{\Theta}=\dot{\Phi}=\frac{\Gamma}{\left(1+\xi \xi^{T}\right)}\left(-\xi^{T} e_{\text {aug }}\right)
$$

where $\Gamma$ is the adaption gain. Since $e_{\text {aug }}$ is chosen so that (16) is SPR, the estimated matrix $\widehat{B}^{-1}=B^{-1}$ is away from singularity and the estimated signals are all bounded, the stability analysis for this adaptive control will yield bounded state $\tilde{x}$ and $y \rightarrow y_{d}$ as $t \rightarrow \infty$ [6].

Remark 2: There might be some problem when applying adaptive control with dynamic extension. The problem occurs if the true decoupling matrix $B(\tilde{x})$ is singular but its estimate $\widehat{B}(\tilde{x})$ is nonsingular during adaptation. In our case, this will not happen since the unknown parameters only appear in the vector fields $\tilde{a}_{1}, \tilde{a}_{2}$ and are not coupled with the state variables. This greatly simplify the computation and the estimated matrix $\widehat{B}=B$ contains no estimate parameters.

\section{Supervisory Switching Control for Ventilation and Stabil- ity Analysis}

Now we start to design the supervisor to decide the recirculation rate $\mu$ such that the $\mathrm{CO}_{2}$ concentration indoor will be adjusted. Note that in [2], the recirculation rate $\mu=75 \%$. Define three classes of the $\mathrm{CO}_{2}$ concentration as $\left\{\mathrm{CO}_{2} \mathrm{High}, \mathrm{CO}_{2} \mathrm{Med}, \mathrm{CO}_{2} \mathrm{Low}\right\}$ where $\mathrm{CO}_{2} \mathrm{High}$ means $C_{s} \geq 1600 \mathrm{ppm}, \mathrm{CO}_{2} \mathrm{Med}$ means $C_{s} \in$ (1200 p pm, $1600 \mathrm{ppm}$ ) and $\mathrm{CO}_{2}$ Low means $C_{s} \leq 1200 \mathrm{ppm}$. The ranges can be defined by requirement. We construct a supervisor $S$ with the following switching logic:

$$
S:\left\{\begin{array}{l}
\mathrm{CO}_{2} \text { High and } y-y_{d}<\varepsilon \Rightarrow \mu=\mu_{3}=60 \% \\
\mathrm{CO}_{2} \text { Med and } y-y_{d}<\varepsilon \Rightarrow \mu=\mu_{2}=65 \% \\
\mathrm{CO}_{2} \text { Low and } y-y_{d}<\varepsilon \Rightarrow \mu=\mu_{1}=75 \% \\
\text { Otherwise }
\end{array}\right.
$$

where $\varepsilon$ is a pre-specified small positive constant. Thus, the hybrid dynamical system (4) is modelled with the continuous states $\tilde{x}$ and discrete states $\mu$ with the continuous state feedback controller $\zeta$ and the discrete state controller $S$. The whole hybrid system model and the processing procedure can be clearly represented by the hybrid automaton [11] shown in Fig. 2.

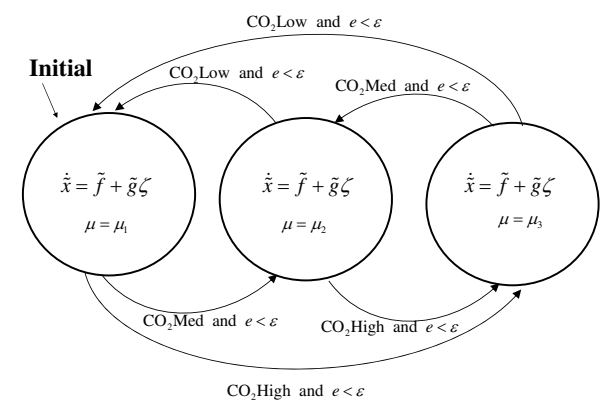

Fig. 2. Hybrid automaton of the HVAC system

Stability issue of this hybrid system will be discussed in the following. Here the stability means that the continuous states are stable and the discrete state will not switch infinite times in finite time interval. Since the value of $C_{s}$ is continuous and slowly time varying, and our switching will only occur when the tracking error of the current system satisfy the specification, the zeno phenomenon [11] will not occur, that is, the existence of the dwell time guarantees that infinitely switching in finite time will never occur.

As discussed in [12], switching between stable systems may lead to unstable phenomenon. Since the continuous dynamical system (8) with adaptive controller $\zeta$ is stable with $\mu=\mu_{i}, i=1,2,3$, in (20), respectively, the possible scenario that leads the system to unstable is that the value of $\mu$ switches sequentially before the continuous states achieves the temperature and humidity tracking and thus oscillation or divergence might occur. The unstable situation caused from consecutive switching is not allowed in the switching mechanism (20) since the supervisor is designed with the priority rule that the switching can only start when the tracking error converges. Hence, we can conclude that the whole system will be stable with the supervisory switching logic. The simplicity of the stability analysis is attributed to our switching logic and the $\mathrm{CO}_{2}$ concentration $C_{S}$ is not coupled with our continuous state dynamic equation $\dot{\tilde{x}}=\tilde{f}+\tilde{g} \zeta$.

\section{Simulation Results}

The equilibrium conditions of the HVAC system are $T_{3}^{e}=$ $71^{\circ} \mathrm{F}, W_{3}^{e}=0.0088 \mathrm{lb} / \mathrm{lb}, \quad T_{0}^{e}=85^{\circ} F, W_{0}^{e}=0.018 \mathrm{lb} / \mathrm{lb}$, $W_{s}^{e}=0.0070 \mathrm{lb} / \mathrm{lb}, u_{1}^{e}=17000 \mathrm{cfm}, u_{2}^{e}=58 \mathrm{gpm}, M_{0}^{e}=$ $166.06 \mathrm{lb} / \mathrm{hr}$, and $Q_{0}^{e}=289897.52 \mathrm{btu} / \mathrm{hr}$. The initial values are $T_{2}^{e}=55^{\circ} \mathrm{F}$ and $\zeta_{1}=15000, \zeta_{2}=40$. Figure 3 shows the first output response of the feedback controller with the design load values $Q_{0}^{e}, M_{0}^{e}$, and the non-design thermal load 
with values $Q_{o}=350000 \mathrm{btu} / \mathrm{hr}$ and $M_{o}=196 \mathrm{lb} / \mathrm{lb}$. It is clear that the controller does not have good tracking performance when the system works upon the environment of non-design load values. The adaptive controller shows its robustness and the transient response is satisfactory compared with [2].

Suppose the values of $Q_{o}, M_{o}$ are changed from 166 to 176 and from 290000 to 310000 , respectively, as shown in the upper side of Fig. 4. In real world, the load changes are in a time scale of hours, here we use these values to show our controller performance. From the bottom of Fig. 4 , the proposed adaptive state feedback controller has shown that it can response to the time varying load change. In Fig. 5, the $\mathrm{CO}_{2}$ concentration changes from $\mathrm{CO}_{2} \mathrm{Low}$ to $\mathrm{CO}_{2} \mathrm{Med}$ at $t=1800(\mathrm{sec})$ and thus the supervisor switches the recirculation rate from $\mu_{1}$ to $\mu_{2}$ at $t=1800$ according to the switching logic. The time response of temperature and humidity ratio shown in Fig. 5 provides the tracking performance with switchings.
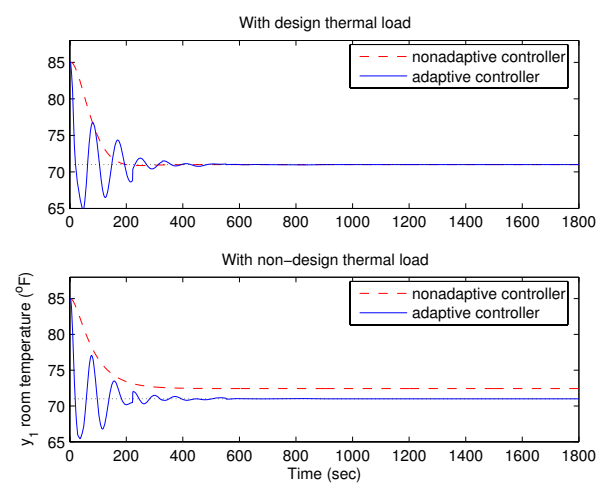

Fig. 3. First output response to design and non-design loads
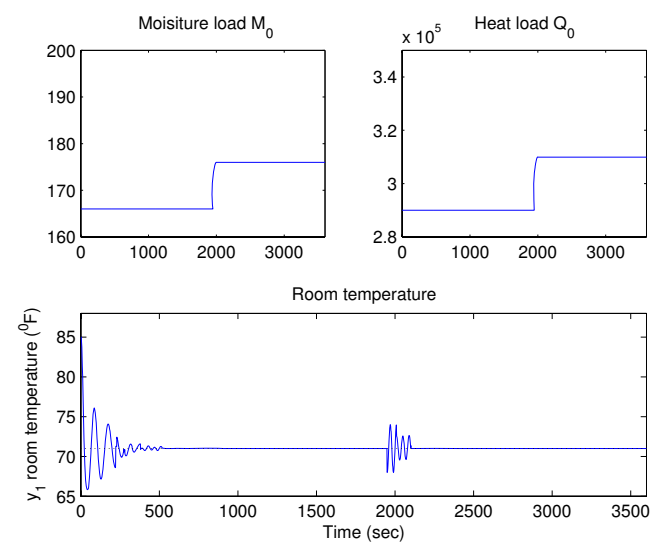

Fig. 4. First output response to time varying $Q_{o}, M_{o}$

\section{CONCLUSIONS}

In this paper, we propose a hybrid system model for the HVAC system and apply the feedback linearization technique with dynamic extension to design the continuous adaptive control for the nonlinear MIMO system. Values of thermal loads $Q_{o}$ and $M_{o}$ may be time varying or not be known
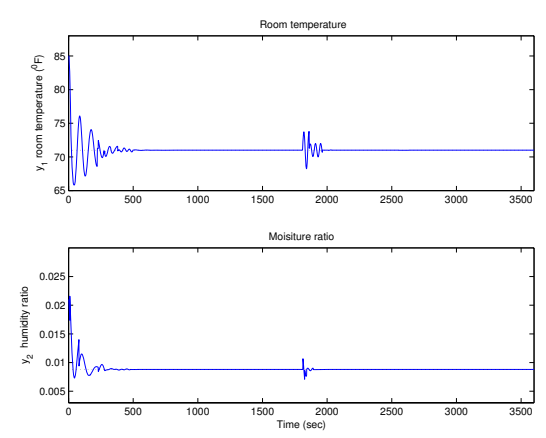

Fig. 5. Output response of the HVAC system with $\mathrm{CO}_{2}$ supervisor.

exactly, and hence adaptive controller is a good choice for HVAC system. Besides, we construct a discrete supervisory controller to adjust the recirculation rate based on the $\mathrm{CO}_{2}$ concentration and discuss stability of the whole system by hybrid system theory. The adaptive controller tracks the desired temperature and humidity ratio to keep the comfort of thermal space and the $\mathrm{CO}_{2}$ supervisor improves the air quality. Computer simulations have proved that such a adaptive controller is superior to the non-adaptive controller due to the ability of adaption to load changes and system perturbation.

\section{REFERENCES}

[1] L. Lu, W. Cai, Y. S. Chai and L. Xie, "Global optimization for overall HVAC systems - Part I problem formulation and analysis," Energy Conversion and management, vol. 46, 2005, pp. 999-1014.

[2] B. Argüello-Serrano and M. Vélez-Reyes, "Nonlinear control of a heating, ventilating, and air conditioning system with thermal load estimation," IEEE Trans. Contr. Syst. Tech., vol. 7, no. 1, pp. 56-63, January 1999.

[3] C. Rentel-Gómez and M. Vélez-Reyes, "Decoupled control of temperature and relative humidity using a variable-air-volume HVAC system and non-interacting control," in Proc. IEEE Int. Conf. on Control Applications, Sept. 2001. pp. 1147-1151.

[4] E. Semsar, M. J. Yazdanpanah, and C. Lucas, "Nonlinear control and disturbance decoupling of an HVAC system via feedback linearization and back-stepping," in Proc. IEEE Int. Conf. on Control Applications, June 2003, pp. 646-650.

[5] Z. Huaguang and L. Cai, "Decentralized nonlinear adaptive control of an HVAC system," IEEE Trans. System, Man, and Cybernetics, Part C: Applications and Reviews, vol. 32, no.4, pp. 493-498, Nov. 2002.

[6] S. S. Sastry and A. Isidori, "Adaptive control of linearizable systems," IEEE Trans. on Automat. Contr., vol. 34, no. 11, pp. 1123-1131, Nov. 1989.

[7] D. Garagić and K. Srinivasan, "Application of nonlinear adaptive control techniques to an electrohydraulic velocity servomechanism," IEEE Trans. on Contr. Syst. Tech., vol. 12, no. 2, pp. 303-314, March 2004.

[8] X. Ma and G. Tao, "Adaptive actuator compensation control with feedback linearization," IEEE Trans. on Automat. Contr, vol.45, no. 9, pp. 1705-1710, Sept. 2000.

[9] X. D. Koutsoukos, P. J. Antsaklis, J. A. Stiver and M. D. Lemmon, "Supervisory Control of Hybrid Systems", Proceedings of the IEEE, vol. 88, no. 7, pp. 1026-1049, 2000.

[10] A. Isidori, "Nonlinear Control Systems," 3rd edition, Springer-Verlag Publication, 1995.

[11] J. Lygeors, K. H. Johansson, S. N. Simić, J. Zhang, and S. S. Sastry, "Dynamical properties of hybrid automata," IEEE Trans. on Automat. Contr., vol. 48, no. 1, pp. 2-17, January 2003.

[12] M. S. Branicky, "Multiple Lyapunov functions and other analysis tools for switched and hybrid systems," IEEE Trans. on Automat. Contr., vol. 43, no. 4, pp. 475-482, April 1998. 